\title{
Sealants recommended to prevent caries
}

\author{
Are pit and fissure sealants effective in preventing decay in children and \\ adolescents who are at risk of caries?
}

\begin{abstract}
Ahovuo-Saloranta A, Hiiri A, Nordblad A, Worthington H, Mäkelä M. Pit and fissure sealants for preventing dental decay in the permanent teeth of children and adolescents (Cochrane Review). In the Cochrane Library. Chichester: John Wiley; 2004, Issue 3.

Data sources Sources of studies were the Cochrane Oral Health Group's Trials Register, the Cochrane Central Register of Controlled Trials, Medline, Embase, Scisearch, SIGLE (System for Information on Grey Literature in Europe) CAplus ${ }^{*}$, INSPEC* ${ }^{*}$, JICST-EPLUS ${ }^{\#}$, NTIS $^{\star}$, PASCAL ${ }^{2 / 3}$, Database of Abstracts and Reviews (DARE), the UK National Health Service Economic Evaluation Database and Health Technology Assessment database. Reference lists from included articles and review articles were searched for additional relevant publications. All relevant studies in most languages were considered and translated.

Study selection Articles were selected for inclusion in the review if they were randomised or quasi-randomised controlled trials of at least 12 months in duration and if sealants were used for preventing caries in children and adolescents of under 20 years of age. Both parallel group and split-mouth study designs were included. The primary outcome was the increment in the numbers of carious occlusal surfaces of premolars and molars.
\end{abstract}

Data extraction and synthesis In the first phase, two reviewers independently examined whether a given study was likely to be relevant on the basis of the title, keywords and abstract. In the second phase, four of the reviewers independently classified whether studies would be included in final analyses. Study authors were contacted for additional information. In the split-mouth studies, relative risk (RR) ratios were calculated for the paired differences of tooth surfaces being carious or not. In studies that compared resin-based sealant with no treatment, fixed-effect meta-analyses were used to combine the estimates of RR ratios. In one parallel-group study, the effect-estimate was calculated from data of occlusal surfaces of teeth included in the test and control groups.

Results Eight trials were included in this review, of which seven were split-mouth studies and one a parallel-group study. Six studies provided data for comparing sealant with no treatment and three studies compared glass ionomers (Gl) with resin-based sealants. The overall effectiveness of resin-based sealants in preventing dental decay on first molars was high. Based on five split-mouth studies with 5-10-year-old children there were significant differences in favour of the secondgeneration resin sealant compared with no treatment. Pooled RR values were $0.14,0.24,0.30$ and 0.43 at $12,24,36$ and $48-54$ months, respectively. The reductions in caries therefore ranged from $86 \%$ at 12 months to $57 \%$ at $48-54$ months. The 24 -month parallel group study that compared second-generation resin sealant with control in 12-13year old children also found significantly more caries in the control group children with a DFS of 0.65 (95\% confidence interval, 0.47 0.83). Allocation concealment was classified as adequate in three of these six studies. The information on background levels of caries in the

Address for correspondence: Emma Tavender, Review Group Co-ordinator, Cochrane Oral Health Group, University Dental Hospital of Manchester, Higher Cambridge Street, Manchester M15 6FH, UK. E-mail: emma.tavender@man.ac.uk. population, however, was insufficient to conduct further analyses that would allow an estimate of the effect of resin-based sealants related to baseline caries prevalence. Only one study provided data for the comparison of $\mathrm{Gl}$ sealant and control. Based on this, there is not enough information to say whether $\mathrm{Gl}$ sealants are effective or not. The results of three studies comparing resin sealants with $\mathrm{Gl}$ sealants were conflicting and the meta-analyses were not carried out.

Conclusions Sealing with resin-based sealants is recommended to prevent caries of the occlusal surfaces of permanent molars but we recommend that the caries prevalence level of both the individual and population should be taken into account. In practice, the benefit of sealing should be considered locally and specific guidelines for clinicians should be used. The methodological quality of published studies concerning pit and fissure sealants was poorer than expected.

${ }^{\ddagger}$ CAplus comprehensive chemistry bibliographic database available from American Chemical Society (CAS). CAplus covers international journals, patents, patent families, technical disclosures, technical reports, books, conference proceedings, and dissertations from all area of chemistry, biochemistry, chemical engineering, and related sciences from 1907 to the present.

*Inspec database formed in 1967, based on the Science Abstracts service which has been provided by the Institution of Electrical Engineers since 1898 providing access to the world's scientific and technical literature in physics, electrical engineering, electronics, communications, control engineering, computers and computing, and information technology.

\#JICST-EPlus is a comprehensive bibliographic database with English citations and abstracts covering the literature published in Japan on all fields of science, technology, and medicine. The file contains indexed and nonindexed records.

^NTIS The National Technical Information Service, part of the US Department of Commerce's Technology Administration, supports the nation's economic growth and job creation by providing access to information that stimulates innovation and discovery.

PASCAL is a unique multidisplinary, multilingual bibliographic database covering the major international literature in science, technology, and medicine. Approximately 5000 journal titles are indexed in PASCAL, which corresponds to a worldwide coverage. The coverage in PASCAL by language is approximately: English $76 \%$, French $10 \%$, Russian $6 \%$, German 5\%, and other languages 3\%.

\section{Commentary}

This is the more rigorous of two current systematic reviews ${ }^{1,2}$ about effectiveness of dental sealants in preventing tooth decay. It is the fourth systematic review on this topic and adds important information to previous extensive reviews (see the Swedish group's 2003 study at www.sbu.se or that by the US National Institutes for Health from 2001 at odp.od.nih.gov/consensus/cons/115/115_intro.htm). As seen in the other reviews, this one shows that in spite of the quantity of articles published on the issue, very few are of optimal methodological quality. A common cause of exclusion was the impossibility of establishing the allocation of patients and the high dropout rates. The first might be solved by better policies at learned journals concerning reporting and peer review of trials, allowing more articles to be analysed. 
In agreement with the review by Mejare et al, ${ }^{1}$ most articles felt worthy of inclusion were conducted in the 1970 s. From a clinical point of view, is it valid to consider the recommendations from an optimal methodological systematic review, of articles of good quality, about materials that are no longer available on the market? In this review studies with first generation sealants were excluded. On the other hand, if it is assumed that we now have access to better materials, we would anticipate that sealants' performance should be better than that of those described. From the researchers' point of view, we can ask ourselves, if we have more materials available now, why do we not have more research than we did 30 years ago? In the future, systematic reviews about therapy with specific materials might improve if they consider the time that passes between the publication of an article and its inclusion in a systematic review and the availability of the therapy or materials.

The strength of this review is that it compares data from different populations such as those of the US, Colombia, New Zealand and Thailand. The analysis infers that sealants have a similar effectiveness regardless of the background levels of caries in each population. Nevertheless the authors take care not to make this inference and ask who benefited following the intervention.

The authors find that all studies have rates of retention higher than $50 \%$ after up to 54 months. The rate of retention is crucial when analysing the effectiveness of resin-based sealants because they are effective only when the tooth is retained. The authors conclude that evidence for resin based sealants is clear. Because only one study provided data for the comparison between GI and control the evidence for sealants based on the GI remains unclear. The authors, however, bring out the possibility that the GI sealants, through their fluoride release, can prevent the development of caries even after the visible loss of sealant material.

\section{Practice points}

- Sealants based on resins are effective for prevention of caries on occlusal surfaces of permanent molars.

- More research is needed to clarify the effectiveness of glass ionomer sealants in caries prevention.

\section{Sergio Uribe}

Department of Preventive and Paediatric Dentistry, Graduate

School, Faculty of Dentistry, University of Valparaiso, Valparaiso, Chile

1. Mejare I, Lingstrom P, Petersson LG, Holm AK, Twetman S, Kallestal C, et al. Cariespreventive effect of fissure sealants: a systematic review. Acta Odontol Scand 2003; 61:321-330.

2. Ahovuo-Saloranta A, Hiiri A, Nordblad A, Worthington H, Mäkelä M. Pit and Fissure Sealants for Preventing Dental Decay in the Permanent Teeth of Children and Adolescents (Cochrane Review). In the Cochrane Library. Chichester: John Wiley; 2004; Issue 3.

Evidence-Based Dentistry (2004) 5, 93-94.

doi:10.1038/sj.ebd.6400292 